\title{
Sparse Principal Axes Statistical Surface Deformation Models for Respiration Analysis and Classification
}

\author{
Jakob Wasza $^{1}$, Sebastian Bauer ${ }^{1}$, Sven Haase ${ }^{1}$, Joachim Hornegger ${ }^{1,2}$ \\ ${ }^{1}$ Pattern Recognition Lab, Department of Computer Science \\ ${ }^{2}$ Erlangen Graduate School in Advanced Optical Technologies (SAOT) \\ Friedrich-Alexander-Universität Erlangen-Nürnberg \\ jakob.wasza@cs.fau.de
}

\begin{abstract}
Detection, analysis and compensation of respiratory motion is a key issue for a variety of medical applications, such as tumor tracking in fractionated radiotherapy. One class of approaches aims for predicting the internal target movement by correlating intra-operatively captured body surface deformations to a pre-operatively learned deformable model. Here, range imaging (RI) devices assume a prominent role for dense and real-time surface acquisition due to their non-intrusive and markerless nature. In this work we present an RI based statistical model built upon sparse principal axes for body surface deformations induced by respiratory motion. In contrast to commonly employed global models based on principal component analysis, we exploit orthomax rotations in order to enable the differentiation between distinctive and local respiratory motion patterns such as thoracic and abdominal breathing. In a case study, we demonstrate our model's capability to capture dense respiration curves and the usage of our model for simulating realistic distinctive respiratory motion patterns.
\end{abstract}

\section{Introduction}

The internal movement of tissue, organs or anatomical structures due to respiratory motion is a crucial problem for a variety of medical applications, such as tomographic reconstruction or fractionated radiotherapy. For the latter, current systems employ gating techniques based on 1-D respiration signals acquired by dedicated sensors attached to the body surface [1] or non-intrusive range imaging (RI) devices [2]. Addressing the low duty cycle of these techniques, current research aims for real-time tumor tracking solutions that re-position the radiation beam dynamically with respect to the internal target motion. Here, the tumor movement is predicted by correlating intra-operatively captured 3-D body surface deformations with a pre-operatively trained 3 -D volumetric model $[3,4,5]$. However, conventional approaches rely on global models obtained from standard principal component analysis that may fail to describe local deformations and that hinder an intuitive interpretation of the model's inherent variations. 

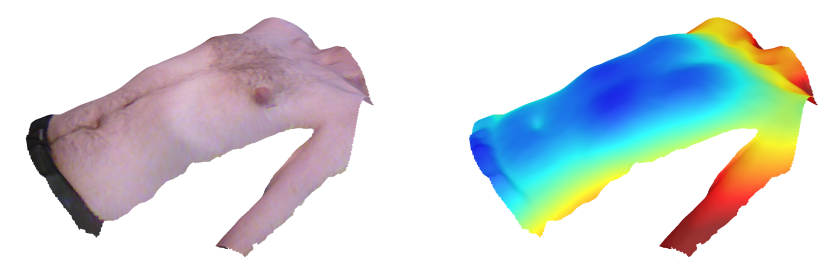

Fig. 1. Body surface of a male subject captured by the Microsoft Kinect RI device. RGB color overlay (left) and color coded depth information (right) where blue and red tones denote closeness and remoteness to the camera, respectively.

In this work, we propose an RI based statistical model for local and distinctive body surface deformations induced by respiratory motion. The basic idea of our approach is to relate local deformations to sparsity of the model's principal axes. Therefore, we build on the orthomax class of statistical methods to derive sparse principal modes of variation. Eventually, this enables the differentiation and intuitive interpretation of local respiratory motion patterns such as thoracic and abdominal breathing. In experiments on real data we demonstrate our model's ability to capture 1-D respiration curves of disjoint anatomical regions without the need to explicitly define the thorax and abdomen regions as proposed in [2]. We further show the usage of our model for simulating patient specific realistic respiratory motion patterns.

\section{Materials and Methods}

The statistical model generation in this work relies on RI devices that deliver dense depth information of the captured body surface in real-time. We denote $f$ as a range image with $f(\boldsymbol{x})$ holding the geometric depth at position $\boldsymbol{x}=(x, y)^{T}$ in the discrete 2-D sensor domain $\Omega: N \times M \mapsto \mathbb{R}$, where $N$ and $M$ denote the image width and height, respectively. Without loss of generality, any 2-D range image $f$ can be linearized as a vector $\boldsymbol{g}$ :

$$
f \equiv \boldsymbol{g}=\left(f\left(\boldsymbol{x}_{1}\right), \ldots, f\left(\boldsymbol{x}_{N \cdot M}\right)\right)^{T}, \quad \boldsymbol{g} \in \mathbb{R}^{N \cdot M} .
$$

We note that this naturally applies for all subregions $\widehat{\Omega} \subseteq \Omega$. A set of linearized RI training frames:

$$
\mathcal{G}=\left\{\boldsymbol{g}_{i}\right\}_{i=1}^{K}, \quad f_{i} \in \widehat{\Omega}
$$

is then used to generate the proposed statistical model using the techniques described in Sec. 2.1 and 2.2. For visualization and analysis purposes, it is often desirable to transform a range image $f$ into a triangulated $3-\mathrm{D}$ point using the intrinsic RI device parameters. Fig. 1 illustrates a body surface acquired from a male subject using an off-the-shelf Microsoft Kinect RI device. 


\subsection{Principal Component Analysis}

Principal component analysis (PCA) is a commonly used technique for the extraction of variations along a given set of training samples and has proven to be a powerful tool in medical image analysis. As a first step, the training set $\mathcal{G}$ is arranged in the configuration matrix $\boldsymbol{L}$ :

$$
\boldsymbol{L}=\left[\boldsymbol{g}_{1}-\overline{\boldsymbol{g}}, \boldsymbol{g}_{2}-\overline{\boldsymbol{g}}, \ldots, \boldsymbol{g}_{K}-\overline{\boldsymbol{g}}\right], \quad \boldsymbol{L} \in \mathbb{R}^{N \cdot M \times K}
$$

with the sample mean $\overline{\boldsymbol{g}}$ defined as $\overline{\boldsymbol{g}}=\frac{1}{K} \sum_{i=1}^{K} \boldsymbol{g}_{i}$. Applying PCA to $\widetilde{\boldsymbol{L}}=\boldsymbol{L}^{T} \boldsymbol{L}$ yields a set of eigenvectors $\left\{\widetilde{\boldsymbol{e}}_{i}\right\}_{i=1}^{K}$ describing the principal modes of variation in the training data as $\boldsymbol{e}_{i}=\boldsymbol{L} \widetilde{\boldsymbol{e}}_{i}, \boldsymbol{e}_{i} \in \mathbb{R}^{N \cdot M}$. The mutually orthogonal modes of variation $\boldsymbol{e}_{i}$ are then sorted in descending order of their respective eigenvalues $\lambda_{i}$. By using the sample mean $\overline{\boldsymbol{g}}$ and the modes that belong to the $P \ll K$ largest eigenvalues, a linear combination of the $P$ principal modes of variation spans a subset of linearized RI frames composed of the given modes of variation:

$$
\boldsymbol{g}^{*}=\bar{g}+\boldsymbol{\Phi} \boldsymbol{b} .
$$

Here, the principal component basis is stored in the matrix $\boldsymbol{\Phi}$ defined as:

$$
\boldsymbol{\Phi}=\left[\boldsymbol{e}_{1}, \boldsymbol{e}_{2}, \ldots, \boldsymbol{e}_{P}\right], \quad \boldsymbol{\Phi} \in \mathbb{R}^{N \cdot M \times P},
$$

and $\boldsymbol{b} \in \mathbb{R}^{P}$ defines the weighting factors for the modes of variation.

We note that PCA yields an orthonormal system that maximizes the variance of the input data along the basis vectors. Hence, applying PCA to the training data usually results in global modes of variation as sparsity in the basis vectors is sacrificed for the sake of variance maximization. By design, this is a crucial drawback with respect to modeling of local and distinctive respiration patterns.

\subsection{Orthomax Rotations}

The orthomax criterion is a technique that is commonly used in factor analysis. Among others, orthomax rotations where successfully employed in sparse modeling of landmark and texture variability [6]. The aim is to transform the global and abstract principal component basis $\boldsymbol{\Phi}$ from Eq. 5 to obtain a more simple structure that can be interpreted in a meaningful manner. The approach in this paper follows the work of [6] and aims to find the orthomax rotation matrix $\boldsymbol{R}_{O} \in \mathbb{R}^{P \times P}$ by solving the constrained optimization problem:

$$
\boldsymbol{R}_{O}=\underset{\boldsymbol{R}}{\operatorname{argmax}} \sum_{j=1}^{P} \sum_{i=1}^{N \cdot M}(\boldsymbol{\Phi} \boldsymbol{R})_{i j}^{4}-\frac{\gamma}{N \cdot M} \sum_{j=1}^{P}\left(\sum_{i=1}^{N \cdot M}(\boldsymbol{\Phi} \boldsymbol{R})_{i j}^{2}\right)^{2},
$$

subject to $\boldsymbol{R}^{T}=\boldsymbol{R}^{-1}$ and $\operatorname{det}(\boldsymbol{R})=1$. Furthermore, $\boldsymbol{R}_{i j}$ denotes the element in the $i^{\text {th }}$ row and $j^{\text {th }}$ column of $\boldsymbol{R}$. The orthomax type is denoted by $\gamma$. This work investigates the varimax rotation only $(\gamma=1)$, thus allowing to solve Eq. 6 by using an iterative scheme employing singular value decompositions [6]. 

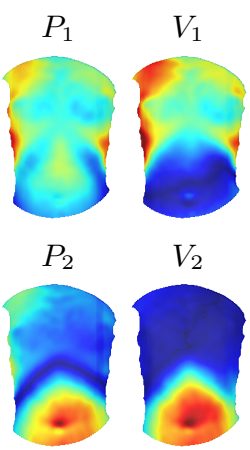

(a) Subject 1
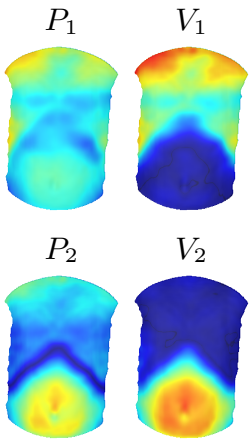

(b) Subject 2

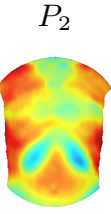

$P_{1}$

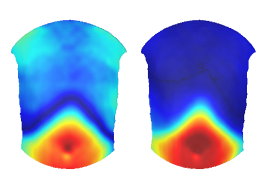

(c) Subject 3

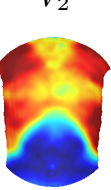

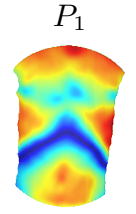

$V_{1}$

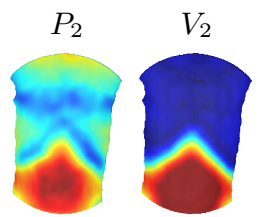

(d) Subject 4

Fig. 2. Modes of variation obtained from standard PCA $(P)$ and varimax rotation $(V)$. The subscripts 1 and 2 denote the first and second principal variation, respectively. The magnitude of variation is color coded from blue (low loadings) to red (high loadings).

To substantiate the use of varimax rotations in this work, we note that $\boldsymbol{R}_{O}$ transforms the principal component basis $\boldsymbol{\Phi}$ from Eq. 5 as $\boldsymbol{\Phi}_{O}=\boldsymbol{\Phi} \boldsymbol{R}_{O}$ in a way that maximizes the variances in the squared variation loadings across all modes. Inherently, this favors sparse modes of variation with several loadings close to zero and others large. This coincides with the aim of this paper to relate local and distinctive respiratory motion patterns to sparsity of the model's modes.

\section{$2.3 \quad$ Experiments}

For our experiments, we captured RI data from four male subjects using a Microsoft Kinect structured light sensor at $30 \mathrm{~Hz}$. To account for sensor noise we perform edge-preserving denoising. The subjects where asked to perform thoracic, abdominal and regular breathing over several respiration cycles. In order to restrict the statistical model to the torso region $\widehat{\Omega}$ only, we applied a fast marching segmentation [8] on the acquired range images. The segmented RI stream of thoracic and abdominal breathing is then sampled with a temporal resolution of $300 \mathrm{~ms}$ to form the training set $\mathcal{G}$ from Eq. 2. We then performed standard PCA as explained in Sec. 2.1 yielding $\Phi$ with the number of modes $P$ chosen such that the model accounts for $\geq 99 \%$ of the total variance in the training data. Finally, as proposed in Sec. 2.2, the sparse varimax model $\Phi_{O}$ is built upon the standard PCA approach.

As application scenarios, we demonstrate our model's capability to simulate selective respiratory motion patterns and to generate 1-D respiration curves of disjoint anatomical regions. For the latter, we project instantaneous RI frames $\boldsymbol{g}$ onto the model's principal axes $\boldsymbol{e}_{i}$ yielding a scalar value $\alpha_{i}=\boldsymbol{g}^{T} \boldsymbol{e}_{i}$ that can be interpreted as a 1-D respiration surrogate. 

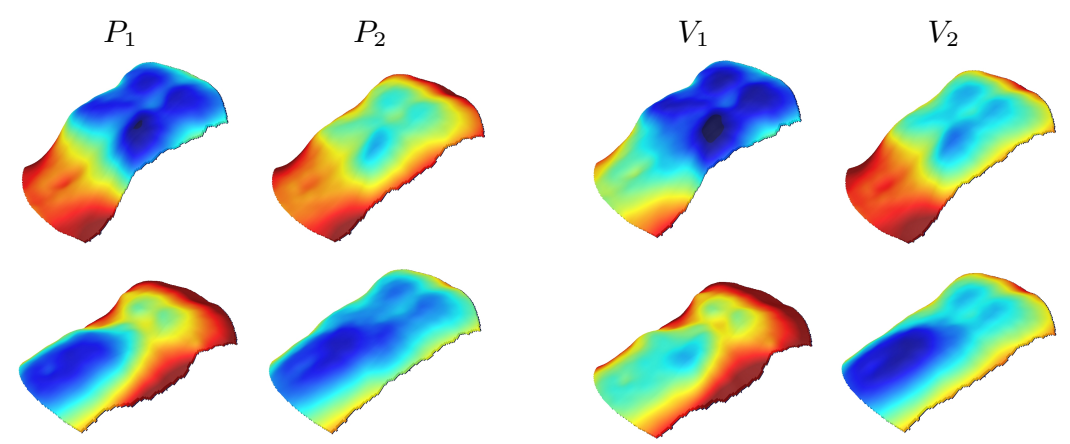

Fig. 3. Effect of varying the modes of variation for Subject 4 . The weights are increased from top $\left(-\sqrt{\lambda_{i}}\right)$ to bottom $\left(+\sqrt{\lambda_{i}}\right)$ for the individual modes. Depth information is color coded where blue and red tones denote closeness and remoteness, respectively.

\section{Results}

For each of the four subjects of this study, two principal modes of variation accounting for $\geq 99 \%$ of the total variance were identified. Fig. 2 depicts the two principal modes of variation as obtained from standard PCA and the varimax model. The PCA based modes exhibit global and meaningless variations, whereas the principal axes obtained from the orthomax model feature local deformations that are highly correlated to thoracic and abdominal breathing, respectively.

Simulated respiratory motion patterns as obtained from our models are depicted in Fig. 3. The varimax model enables the selective simulation of thoracic and abdominal breathing whereas deformations obtained from standard PCA are of no relevance with respect to the human respiration system.

Respiration curves generated with our models are illustrated in Fig. 4. For abdominal breathing, the first mode obtained from the orthomax model exhibits a smaller total variation and amplitude compared to the standard PCA approach. A similar behavior is observable for thoracic breathing and the second mode. This indicates that a varimax model is better suited for analysis of local and disjunct motion patterns. With regard to regular breathing, the PCA modes are not interpretable in an intuitive manner. In contrast, the varimax modes indicate that the subject mainly performed abdominal respiration with some thoracic movement superimposed.

\section{Discussion}

We have shown that the orthomax class of statistical methods can be used to generate a model for local surface deformations induced by respiratory motion. Our experimental study revealed that, in contrast to a standard PCA approach, distinctive and local respiratory motion patterns can be analyzed and classified. 


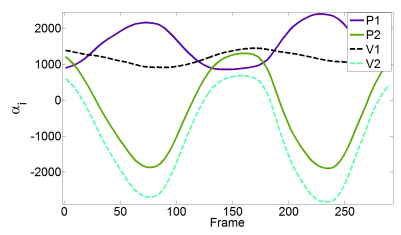

(a) Abdominal

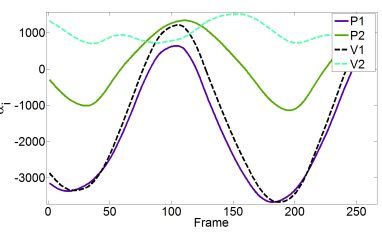

(b) Thoracic

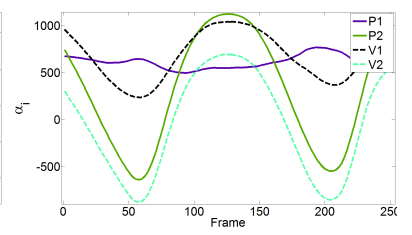

(c) Regular

Fig. 4. Using the statistical model to generate 1-D respiration curves. For the varimax model, the first principal mode accounts for thoracic and the second mode for abdominal breathing.

However, we note that orthomax methods only represent a small class of statistical methods for respiration analysis. Therefore, ongoing work investigates alternative statistical methods and non-linear techniques for model generation.

\section{Acknowledgments}

J. Wasza and S. Bauer gratefully acknowledge the support by the European Regional Development Fund (ERDF) and the Bayerisches Staatsministerium für Wirtschaft, Infrastruktur, Verkehr und Technologie (StMWIVT), in the context of the R\&D program IuK Bayern under Grant No. IUK338. S. Haase is supported by the Deutsche Forschungsgemeinschaft (DFG) under Grant No. HO 1791/7-1.

\section{References}

1. Keall PJ, Mageras GS, Balter JM, Emery RS, Forster KM, Jiang SB, et al. The management of respiratory motion in radiation oncology, report of AAPM Task Group 76. Med Phys. 2006;33(10):3874-3900.

2. Müller K, Schaller C, Penne J, Hornegger J. Surface-based Respiratory Motion Classification and Verification. In: Proc BVM; 2009. p. 257-261.

3. Fayad H, Pan T, Roux C, Le Rest CC, Pradier O, Clement JF, et al. A patient specific respiratory model based on $4 \mathrm{D}$ CT data and a time of flight camera (TOF). In: Proc IEEE NSS/MIC; 2009. p. 2594-2598.

4. Hoogeman M, Prévost JB, Nuyttens J, Pöll J, Levendag P, Heijmen B. Clinical accuracy of the respiratory tumor tracking system of the cyberknife: assessment by analysis of log files. Int J Radiat Oncol Biol Phys. 2009;74(1):297-303.

5. Ehrhardt J, Werner R, Schmidt-Richberg A, Handels H. Statistical Modeling of 4D Respiratory Lung Motion Using Diffeomorphic Image Registration. IEEE Trans Med Imaging. 2011;30(2):251-265.

6. Stegmann MB, Sjöstrand K, Larsen R. Sparse Modeling of Landmark and Texture Variability using the Orthomax Criterion. In: Proc SPIE. vol. 6144; 2006. p. 61441G1-61441G.12.

7. Sethian JA. Level Set Methods and Fast Marching Methods. 2nd ed. Cambridge University Press; 1999. 